\title{
Suitable MOTHER WAVELET SELECTION FOR EEg SIGNALS ANALYSIS: FREQUENCY BANDS DECOMPOSITION AND DISCRIMINATIVE FEATURE SELECTION
}

\author{
Romain Atangana ${ }^{\mathbf{1 , 2 , 3}}$, Daniel Tchiotsop ${ }^{\mathbf{1}}$, GodpromesseKenne ${ }^{\mathbf{1}}$, Laurent Chanel \\ Djoufack Nkengfack ${ }^{\mathbf{1 , 2}}$ \\ ${ }^{1}$ Unité de Recherche d'Automatique et d'InformatiqueAppliquée (UR-AIA), IUT-FV of \\ Bandjoun, University of Dschang-Cameroon, P.O. Box 134-Bandjoun \\ ${ }^{2}$ Unité de Recherche de Matière Condensée-d'Électronique et de Traitement du Signal \\ (UR-MACETS), Faculty of Science, University of Dschang-Cameroon, P.O. Box 67- \\ Dschang \\ ${ }^{3}$ Division of Continuing Education and Distance Learning, Higher Teacher Training \\ College, University of Ngaoundere-Cameroon, P.O. Box 652-Bertoua
}

\begin{abstract}
Wavelet transform (WT) is a powerful modern tool for time-frequency analysis of non-stationary signals such as electroencephalogram (EEG). The aim of this study is to choose the best and suitable mother wavelet function (MWT) for analyzing normal, seizure-free and seizured EEG signals. Several MWTs can be used, but the best MWT is the one that conserves the quasi-totality of information of the original signal on wavelet coefficients and gather more EEG rhythms in terms of frequency. In this study, Daubechies, Symlets and Coiflets orthogonal families were used as bsis mother wavelet functions. The percentage rootmeans square difference (PRD), the signal to noise ratio (SNR) and the simulated frequencies as the selection metrics. Simulation results indicate Daubechies wavelet at level $4(D b 4)$ as the most suitable MWT for EEG frequency bands decomposition.Furthermore, due to the redundancy of the extracted features, linear discriminant analysis (LDA) is applied for feature selection. Scatter plot showed that the selected feature vector represents the amount of changes in frequency distribution and carries most of the discriminative and representative information about their classes. Then, this study can provide a reference for the selection of a suitable MWT and discriminativefeatures.
\end{abstract}

\section{KEYWORDS}

Electroencephalogram (EEG),Mother Wavelet (MWT), Frequency Bands Decomposition,Features Selection, Linear Discriminant Analysis (LDA)

\section{INTRODUCTION}

Exploring the brain's electrical activities using electroencephalogram (EEG) signals has increased recently. EEG is a powerful technique to testthe brain function and its activities. EEGs have great potential for the diagnosis and treatment of neurobiological disorders, mental and brain diseases such as epilepsy, sleep disorders, coma, encephalopathy and brain death [1]. Diagnostic applications generally focus on the spectral content of EEGs, that is, the type of neural oscillations that can be observed in EEG signalssincetheir frequency content provides useful information than time-domain representation [2].EEG signals commonly include five neural 
Signal \& Image Processing: An International Journal (SIPIJ) Vol.11, No.1, February 2020

oscillations or frequency bands namely (from lower to higher frequencies) : Delta $(0.5-4 \mathrm{~Hz})$, Theta (4-8 Hz), Alpha $(8-13 \mathrm{~Hz})$, Beta $(13-30 \mathrm{~Hz})$ and Gamma $(30-60 \mathrm{~Hz})$.

Recently, many signal analysis methods have been proposed to decompose EEG signals into frequency bands, extract and selectfeatures for their analysis and classification. In this impetus, Pushpendra et al. [3], proposed an analysis process based on the Fast Fourier Transform (FFT) technique.This method does not provide correlation between the time localization and the frequency changes since the time-frequency information is not directly obtained from the coefficients produced by the FT.Ji Zhong et al. [4], Wu and Huang [5], and Pachori and Bajaj [6] exploited the empirical mode decomposition (EMD) for frequency bands separation. EMD is an estimation method to separate a signal into a series of intrinsic signals based on the calculation of the local extremum and from the calculation of stream cubic extrapolation. EMD technique provides two envelops and their difference defines an intrinsic function. Also, Pradip and Ram [7]usedthe general three terms equation to approximate the frequency bands associated to discrete orthogonal polynomials and separate EEG frequency bands. During this last decade, the useful wavelet transforms (WT) remains one of the most used methodsfor the purpose of EEGfrequency bands separation [8-16]. WT is a powerful spectral estimation technique for the time-frequency analysis of non-stationary signals, such as EEGs. WT is defined as a set of decomposition functions of the correlation between the signal and the shifting and dilating of one specific function calledmother wavelet function (MWT). In most cases, appropriateMWT and the decomposition level are selected on the basis of their compatibility with the EEG signal characteristics to be analyzed. However, several common standard wavelet families, including Daubechies, Symlets and Coiflets wavelets are considered [17-19]. Therefore, for informative feature selection, the critical point in EEG signal processing via WT remains the selection of the decomposition level and the suitable MWT.

In signals analysis using WT, a typical way is to inspect the data visually. The features extracted from the waveforms, such as durations slope, sharpness and amplitude form the basis for the mimetic methods. But, the signal is commonly analyzed by investigating frequency bands related to various conscious states or mental activities [14]. Most often, tests are performed with different types of wavelets and the one which gives maximum efficiency is selected for the particular application. Raw EEG signals have much data, but not much information. Then, the input data needs to be transformed into a reduced representation set of features. In automatic EEG signals analysis, the relevance of feature extraction and selectionishighlighted[20].Therefore, to ensure both the performance of the diagnosis system and compensate the loss of information, extraction and selection of parameters via dimensionality reductionis necessary since informative features are those with the greatest possible discriminative ability. In this paper we intend to use WT with suitable MWT to decompose raw EEG signals into theirfive clinical frequency bands and select some useful informative and discriminative featuresbasedon wavelet coefficients. For this purpose, 45 orthogonal MWTs, statistics features, entropy feature, energy features, and LDA are used.

The second section of this paperfirst presents the EEG data sets used and the WT analysis method. Secondly, the selection scheme of the suitable MWT for wavelet analysis is constructed and exploited for the extraction and selection of discriminative features using LDA. In the third section, simulation results and discussions are presented. Then, the last section summarizes the study with some concluding remarks. 
Signal \& Image Processing: An International Journal (SIPIJ) Vol.11, No.1, February 2020

\section{RELATED WORKS}

Zaid Abdi and al. (2017) [21] proposed the use of various mother wavelet function to find the efficient signal denoising. The mother wavelet function which are selected are db4, Sym7, Coif3, and Bior3.9. For evaluating the efficient mother wavelet function EEG signal was corrupted using three different noises which are Power line noise (PLN), (EMG) and white Gaussian noise (WGN), the denoised EEG is evaluated using five measurement factors. SNR, SNR improvement, MSE, RMSE, and the PRD. Finally, Coif3 filter achieved the efficient EEG signal denoising for PLN and EMG noise, Sym7 filter obtained the best result with WGN.

Al-QAZZAZ and al [17] performed a comparative study to select the efficient mother wavelet basis function that optimally represent the signal characteristics of the electrical activity of the human brain during a working memory task recorded through electroencephalography. Daubechie (db1-db20), Symlets (sym1-sym20), and Coiflet (Coif1-Coif5) were evaluated based on the cross-correlation with the EEG signal. The best MWT basis function is the one with the most significant differences to the task concerned and to maximize the cross-correlation with the EEG signal.

Mohd T. and al [19] work to recognize the best and suitable wavelet family for analyzing cognitive memory using EEG signal. The criteria of the EEG signal were narrowed down to the Daubechies, Symlets and Coiflets and the final selection depend on the Means Square Error (MSE). To analyze EEG, signal the MSE must be low. The result showed that db4 had a low MSE, though the MSE for Coif5 was lower, the difference between compressed and original EEG signal were higher, so it was postulated that some information was missing.

In the work of Al-kadi and al. (2012) [32]one hundred and thirteen potential mother wavelet function amount which (Daubechies, Coiflet, Biortogonal, Reverse Biorthogonal, Discrete Meyer, and Symlets) are selected and investigate to find the most similar function with EEG signal. The mother wavelet that most compatible with EEG signal has been founded by determines the minimum means square error (MSE) and the larger signal - to- noise ratio (SNR). Both values showed that the compatibility of the mother wavelet symlet (sym24) for denoising is the best.

In the work of Rafiee J. and al. in 2011[31] on wavelet basis function in biomedical signal processing, the outcome of the correlation between signal and continuous wavelet basis function (CWC) has been established as the basis for comparing selected mother wavelet functions. The result show that from Daubechies family, db44 is the most similar function for all cases studies.

Duo Chen and al. [22] used seven most used wavelet families to make test through full level of decomposition. The maximum level of decomposition is jointly determined by the signal and the mother wavelet to satisfy the criteria. Finally, the finding establishes that wavelet coefficient at each band can be used to construct feature vector for the EEG signal. Therefore, deeper decomposition means more frequency bands and thus longer feature vectors. An optimal level of decomposition should provide a good balance between computation complexity and accuracy.

Ning Ji and al. in 2019 [23] worked on "Appropriate mother wavelet for continuous Gait Event Detection Based on time - frequency analysis for Hemiplegic and Healthy Individuals". In order to determine an appropriate mother, they systematically investigated the performance of 32 commonly applied mother wavelets in detecting gait events using the measures of both accuracy and quantitative criteria. The mother wavelet that provides the maximum time-error is generally considered to be the most appropriate mother wavelet for gait event detection. The findings 
Signal \& Image Processing: An International Journal (SIPIJ) Vol.11, No.1, February 2020 suggested that the "db6" is an appropriate mother wavelet in gait event detection for both healthy and hemiplegic subject:

\section{Materials And Methodology}

\section{1 . EEG Data Sets}

Most researchersin the field of EEG signal analysis use the publicly available data described in [24]. The data was collected from ten patients and consists of five sets(A-E)each containing 100 EEG segments recorded through single channel.The first two sets (SET A and SET B) consist of data collected from five healthy volunteers, relaxed in an awake state with eyes open for A and closed for B. SET C segments have been measured intracranial in seizure-free intervals from five others patients in the epileptogenic zone, and SET D from the hippocampal formation of the opposite hemisphere of the brain of the same patients in seizure-free intervals. SET E consists of data recorded intracranial during a seizure.The data set were recorded using a 128-channel amplifier system and standardized 10-20 electrode placement scheme. Knowing that the duration of the record is 23.60 seconds with a sampling frequency of $173.61 \mathrm{~Hz}$, the bandwidth of the signal will be extended from $0.045 \mathrm{~Hz}$ to $86.6 \mathrm{~Hz}$. As useful information from the data can be found only in $\delta, \theta, \alpha, \beta$, and $\gamma$ sub-bands, a band pass filter with $0.50-40 \mathrm{~Hz}(12 \mathrm{Db} / \mathrm{oct})$ was applied.Figure1 below presents examples of EEG signals of the data sets.

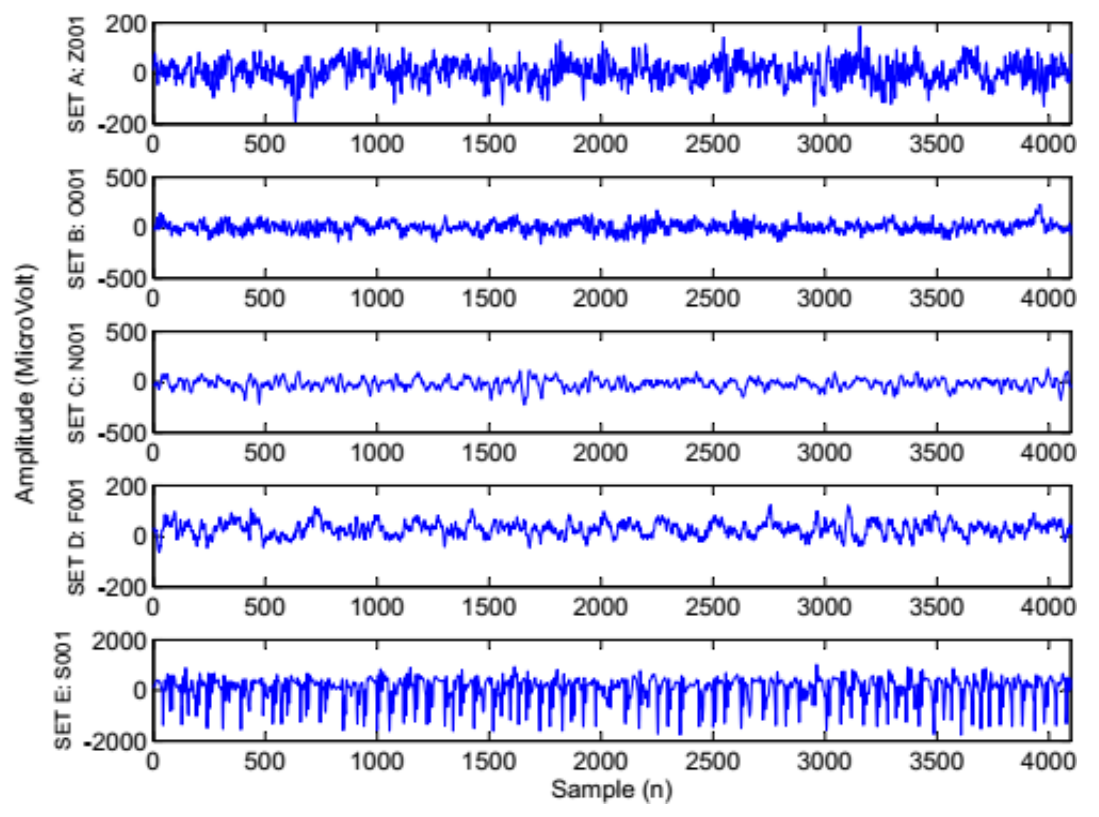

Figure 1: Examples of EEG signals[21].

\subsection{Wavelet Analysis}

WT is an effective processing method introduced to address the problem of time-frequency analysis of non-stationary signals [25]. The continuous WT (CWT) of a signal $x(t)$ is defined as:

$$
C W T_{a, b}(x)=\frac{1}{\sqrt{|a|}} \int x(t) \psi^{*}\left(\frac{t-b}{a}\right) d t
$$


Signal \& Image Processing: An International Journal (SIPIJ) Vol.11, No.1, February 2020

Where $\psi(t)$ is called the mother wavelet function (MWT), while $a$ and $b(a, b \in I R)$ are dilation and translation parameters, respectively.

The variable $a$ plays the role of the reverse of the frequency, therefore the more $a$ is small, the less the MWT is extended in time domain and the more the central frequency of the spectrum is higher. We can also interpret this expression as the projection of the signal whenanalyzing family functions $\psi_{a, b}$ constructed from the mother wavelet function $\psi$ in conformity to the following equation:

$$
\psi_{a, b}(t)=\frac{1}{\sqrt{a}} \psi\left(\frac{t-b}{a}\right)
$$

According to Daubechies [25], if the mother wavelet function is well chosen, then the wavelet transform is reversible, and the signal can be reconstructed after analysis and the synthesis technique through the following equation:

$$
x=C_{\psi}^{-1} \int_{-\infty}^{+\infty} \int_{-\infty}^{+\infty} \frac{1}{a^{2}}\left\langle x, \psi_{a, b}\right\rangle \psi_{a, b} d a d b
$$

This is only possible in theory because the computation is only possible numerically and the convergence can be too slow. If the coefficient $C_{\psi}$ exists, it can be written as:

$$
C_{\psi}=2 \pi \int_{-\infty}^{+\infty}|\psi(\omega)|^{2} \frac{d \omega}{\omega}
$$

The existing condition of this coefficient is equally due to the admissibility condition of the mother wavelet function. This condition is expressed through the following equation.

$$
\int_{0}^{+\infty}|\psi(\omega)|^{2} \frac{d \omega}{\omega}=\int_{-\infty}^{0}|\psi(\omega)|^{2} \frac{d \omega}{\omega} \prec \infty
$$

This relation is usually related to the condition expressed on equation (6) which is not too constraint and indicate that the mother wavelet function must only be of average value equal to zero.

$$
\int_{-\infty}^{+\infty} \psi(t) d t=0
$$

The application of WT in engineering fields usually requires the discrete WT (DWT). DWT, which is non-redundant and has less computational time than CWT. DWT is defined by using the discrete values of the parameters $a$ and $b$.

$$
D W T_{m, n}(x)=a_{0}^{-m / 2} \int x(t) \psi\left(a_{0}^{-m / 2} t-n b_{0}\right) d t
$$

Where $m$ and $n(m, n \in Z)$ are indicating frequency and time localization, respectively. 
Signal \& Image Processing: An International Journal (SIPIJ) Vol.11, No.1, February 2020

Mallat[26] developed a dyadic method by which DWT separates a signal into low and highfrequency components via two finite impulse response quadratic mirror filters. More details of the dyadic-orthonormal WT can be found in [30]. The result transforms the original signal of dominant frequency $F_{N}$ into two equal sub-bands $\left[0-F_{N / 2}\right]$ and $\left[F_{N / 2}-F_{N}\right]$ and the highfrequency component can be sent to the second stage to repeat the procedure as depicted by Figure 2. Finally, the signal is decomposed at the expected level in terms of detail and approximation coefficients.

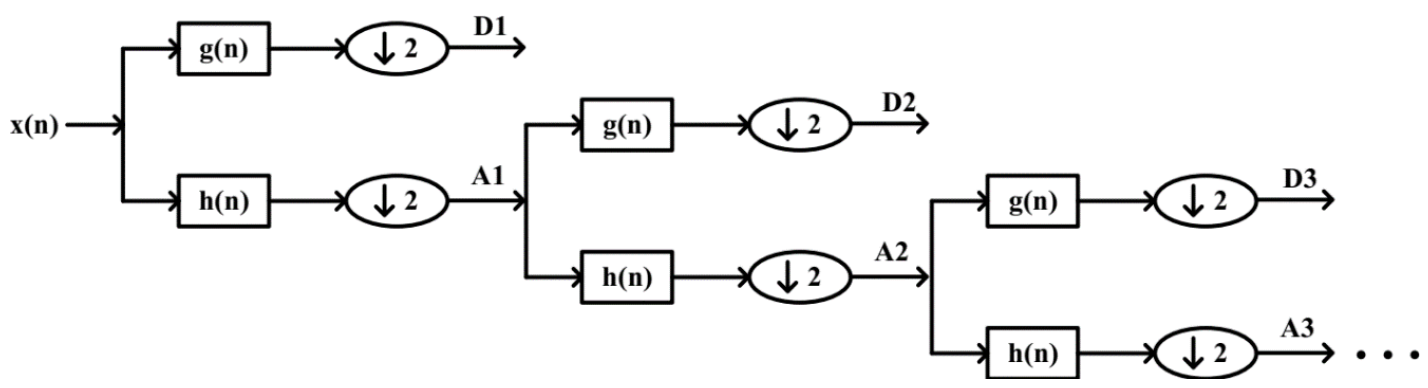

Figure 2: Frequency bands decomposition using WT.

\subsection{Mother Wavelet Selection For Frequency Bandsdecomposition}

The five primary EEG sub-bands generally span the $0.5-60 \mathrm{~Hz}$ frequency range and higher frequencies are often characterized as noise. The details and approximationscoefficients individually hold a quantity of the energy of the signal. It is left to determine the decomposition level and the suitable MWT which better decomposeEEG into its different frequency bands.

Generally, decomposition level and optimal MWT function are selected based on the compatibility with the EEG signal characteristics to be analyzed. Decomposition level is selected with respect to the dyadic decomposition tree and is guided by the dominant frequency. The MWT and the wavelet decomposition level should be chosen carefully when WT-based processing methods are used. The choice of the MWT is then an open principle, but we must know the power and the speed of convergence of the algorithm of the reconstructed signal are too dependent on the choice of the MWT. At the end, the wavelet transform will be interesting as signal analysis tool only if the MWT remains well localized in time and in frequency respectively. Also, accurate MWT selection not only helps to retain the original signal but can also reduce overlapping that appears on the different sub-bands. In order to ensure the optimal reconstruction of the analyzed signal, only45 MWTs from three different orthogonal families, including Daubechies (Db1-Db20), Symlets (sym1-sym20), and Coiflets (coif1-coif5) are used $[17,19]$. These MWTs are regarded as the most common parameters in biomedical signal processing since they are built to conserve the quasi-totality of information of the original signal on wavelet coefficients [17,27-28]. An important concern about the extraction of features based on WT is that the quality of the features depends highly on MWT form namely the basis function that best correlates to the seizure's morphology and the signal frequency components. Many authors pointed out that the wavelet basis function choice is critical to achieve relevant features [29-30].

The quality of reconstruction of the analyzed normal, seizure-free and seizure EEG signals, and consequently the choice of the suitable MWT are evaluated using the simulated frequency, the percentage root mean square difference (PRD) and the signal to noise ratio (SNR) defined by equations (8) and (9), respectively. The PRD is the most prominently used distortion measure defined as the average quadratic error. The SNR is a measure used to compare the level of a desired signal to the level of background noise. 


$$
\operatorname{PRD}(\%)=100 * \sqrt{\frac{\sum_{n=1}^{N}\left(x_{o}(n)-x_{a}^{j}(n)\right)^{2}}{\sum_{n=1}^{N}\left(x_{o}(n)-\overline{x_{o}}(n)\right)^{2}}}
$$

Where $\overline{x_{o}}(n)$ denotes the mean value of the original signal $x_{o}(n)$, and $x_{a}^{j}(n)$ represents the $j^{\text {th }}$ approximate signal.

$$
S N R=10 \log \left|\frac{\sum_{n=1}^{N}\left(x_{o}(n)-\overline{x_{o}}(n)\right)^{2}}{\sum_{n=1}^{N}\left(x_{o}(n)-x_{a}^{j}(n)\right)^{2}}\right|=-20 \log (P R D)
$$

\subsection{Feature Extraction}

In pattern recognition, feature extraction is a special form of dimensionality reduction. When the input data to an algorithm is too large to be processed and it is suspected to be notoriously redundant that is much data but not much information, then the input data will be transformed into a reduced representation set of features also named feature vector.The extraction methodology analyzes signals to extract further information from the raw signal.The extracted wavelet coefficients provide a compact representation that shows the energy distribution of the EEG signal in time and frequency domains. In order to further reduce the dimensionality of the extracted feature vectors, typical statistics, measure of randomness andenergy featuresin each frequency band are computed based on previously derived wavelet coefficients $[9,16]$. Then, the time-frequency distribution of the EEG signals can be represented by the following features:

- Maximum (Max) of the absolute values of the wavelet coefficients in each frequency band.

- Mean (Mean) of the absolute values of the wavelet coefficients in each frequency band.

- Standard deviation (StD1) of the wavelet coefficients in each frequency band.

- Standard deviation (StD2) of the absolute values of the wavelet coefficients in each frequency band.

- Entropy of the absolute values of the wavelet coefficients in each frequency band. For the frequency band with wavelet coefficients $\alpha_{j}$, it is expressed as:

$$
S=\sum_{k}\left|\alpha_{j}\right| \log \left(\left|\alpha_{j}(k)\right|^{2}\right)
$$

- Energy of the original EEG signal.

\subsection{Featuresselection Using Linear Discriminant Analysis (LDA)}

After processing the data with WT,LDA analyzes the statistics, entropy and energy features to select the most prominent featuresthat are representative of the various classes of signals. The main aim of LDA features selection is to obtain further information from the signal by transforming itstypical statistics, entropy and energyfeaturesinto fewer ones asfeature vectors. 
Signal \& Image Processing: An International Journal (SIPIJ) Vol.11, No.1, February 2020

The selected features are more informative and discriminative, having lessnoise and redundancy with respect to the extracted ones.

LDA is a supervised algorithm since it computes the linear discriminant features by maximizing distance between classes and minimizing distance within classes [31]. The LDA projection can be written as a single matrix equation described by:

$$
Y=X * W
$$

Where $\mathrm{Y}$ and $\mathrm{X}$ are the original data and features, respectively. And $W$ is the $d x k$ projection matrix formed from the first $k$-eigenvectors of $S_{w}^{-1} S_{b}$, where $S_{w}$ and $S_{b}$ is the within-class and between-class scatter matrices, respectively.

$$
\begin{aligned}
& S_{w}=\frac{1}{N} \sum_{l=1}^{L} \sum_{i=1}^{N_{l}}\left(x_{i}^{l}-\mu_{l}\right)^{T}\left(x_{i}^{l}-\mu_{l}\right) \\
& S_{b}=\frac{1}{N} \sum_{l=1}^{L} N_{l}\left(\mu_{l}-\mu\right)^{T}\left(\mu_{l}-\mu\right)
\end{aligned}
$$

$x_{i}^{l}, N_{l}$ and $\mu_{l}$ are an $i$-row vector, the number of $i$-vectors and the mean vector corresponding to class label $l$, respectively. $L, N$ and $\mu$ are the number of classes, the total number of $i$-vectors and the global mean of all classes, respectively.

\section{RESUlTS AND DISCUSSION}

This section presents simulation results and discussion. All simulations are carried out using the MATLAB R2013a software.In this study, the entire database is used to perform different features extraction and selection.

\subsection{MWT Selection Andfrequency Bands Decomposition Using WT}

Choosing the suitable MWT is more crucial for a successful WT frequency bands decomposition. Then, before extracting useful information from the EEG signals, WT must be applied to all sets of EEG signals in order to select the suitable MWT for the purpose. The current EEG signals are first pre-processed in order to fix the dominant frequency before applying WT. For the purpose, an ordered 30 low-pass FIR filter is designed with $3 \mathrm{Db}$ of ripple in the passband from 0 to $64 \mathrm{~Hz}$ and at least $60 \mathrm{Db}$ of attenuation in the stop-band. Since the filtered EEG signals have a dominant frequency of $64 \mathrm{~Hz}$, this imposes a four-level decomposition using DWT. The value 64, which is an exact power of two, is used instead of $60 \mathrm{~Hz}$, to obtain more easily the frequency bands of the EEG during the wavelet analysis.Different types of MWT of different orders including Daubechies (Db1 to Db20), Symlets (Sym1 to Sym20), Coiflets (Coif1 to Coif5) are used to analyze EEGs signal. The choice is guided from the ability of the above three different orthogonal families of MWT to reproduce the EEG output signal during reconstruction after the synthesis and also the ability that permits to specify the characteristics of the signal in time and frequency domains respectively.These abilities are examined in terms of simulated frequency, PRD and SNR, are presented in Table 1, Table 2 and Table 3. The MWT which presents a minimum PRD and a maximum SNR with simulated frequency as stated by the DWT method is chosen as suitable MWT to perform the analysis and the synthesis of EEG signals. This MWT is prone to 
Signal \& Image Processing: An International Journal (SIPIJ) Vol.11, No.1, February 2020 give better performances during sub-bands frequency decomposition for feature extraction and selection.

Table 1: Comparison of the simulated frequency in Delta, Theta and Alpha rhythms for the 45 MWTs.

\begin{tabular}{|c|c|c|c|c|c|c|c|c|c|c|c|c|c|c|c|}
\hline \multirow{2}{*}{ MWT } & \multicolumn{5}{|c|}{ Simulated frequencr in Delta rhythm $(\mathrm{Hz})$} & \multicolumn{5}{|c|}{ Simulated frequencr in Theta rhythm $(\mathrm{Hz})$} & \multicolumn{5}{|c|}{ Simulated frequencr in Alpha rhythm (Hz) } \\
\hline & SET A & SET B & SET C & SET D & SET E & SET A & SET B & SET C & SET D & SET E & SET A & SET B & SET C & SET D & SET E \\
\hline Dbl & 0.20 & 0.29 & 0.32 & 0.37 & 2.16 & 8.24 & 9.47 & 7.25 & 7.30 & 6.86 & 12.98 & 12.22 & 15.04 & 15.37 & 14.89 \\
\hline $\mathrm{Db} 2$ & 0.21 & 0.33 & 0.35 & 0.38 & 2.45 & 8.30 & 9.53 & 6.98 & 6.94 & 6.88 & 12.57 & 12.24 & 13.33 & 13.77 & 13.58 \\
\hline $\mathrm{Db} 3$ & 0.22 & 0.30 & 0.36 & 0.43 & 2.62 & 7.97 & 9.35 & 6.79 & 6.84 & 6.92 & 12.85 & 12.30 & 13.09 & 13.17 & 13.44 \\
\hline $\mathrm{Db} 4$ & 0.22 & 0.33 & 0.36 & 0.43 & 2.80 & 8.12 & 9.40 & 6.82 & 6.91 & 6.85 & 12.69 & 12.25 & 13.11 & 13.11 & 13.24 \\
\hline $\mathrm{Db} 5$ & 0.22 & 0.35 & 0.36 & 0.40 & 2.68 & 8.03 & 9.40 & 6.73 & 6.83 & 6.78 & 12.77 & 12.33 & 13.06 & 12.93 & 13.19 \\
\hline $\mathrm{Db} 6$ & 0.22 & 0.35 & 0.36 & 0.42 & 2.74 & 7.95 & 9.35 & 6.79 & 6.92 & 6.79 & 12.68 & 12.27 & 12.92 & 12.78 & 13.20 \\
\hline $\mathrm{Db} 7$ & 0.22 & 0.35 & 0.36 & 0.42 & 2.87 & 7.99 & 9.33 & 6.83 & 6.85 & 6.80 & 12.73 & 12.28 & 12.98 & 12.64 & 13.22 \\
\hline $\mathrm{Db} 8$ & 0.22 & 0.35 & 0.39 & 0.42 & 2.74 & 7.94 & 9.25 & 6.71 & 6.80 & 6.94 & 12.68 & 12.28 & 12.92 & 12.84 & 13.31 \\
\hline $\mathrm{Db} 9$ & 0.22 & 0.34 & 0.36 & 0.42 & 2.86 & 7.90 & 9.26 & 6.84 & 6.87 & 6.93 & 12.82 & 12.26 & 12.88 & 12.48 & 13.05 \\
\hline Dblo & 0.22 & 0.34 & 0.36 & 0.42 & 2.86 & 7.94 & 9.15 & 6.77 & 6.84 & 6.86 & 12.68 & 12.27 & 12.97 & 12.87 & 13.43 \\
\hline Dbll & 0.22 & 0.34 & 0.39 & 0.42 & 2.81 & 8.00 & 9.35 & 6.68 & 6.79 & 6.88 & 12.64 & 12.28 & 12.72 & 12.61 & 13.15 \\
\hline $\mathrm{Db} 12$ & 0.22 & 0.34 & 0.36 & 0.42 & 2.87 & 7.86 & 9.24 & 6.92 & 6.90 & 6.87 & 12.63 & 12.28 & 12.81 & 12.70 & 13.33 \\
\hline $\mathrm{Dbl3}$ & 0.22 & 0.35 & 0.36 & 0.42 & 2.82 & 8.11 & 9.35 & 6.76 & 6.87 & 6.90 & 12.68 & 12.31 & 12.71 & 12.65 & 13.17 \\
\hline $\mathrm{Dbl4}$ & 0.22 & 0.35 & 0.39 & 0.42 & 2.82 & 7.87 & 9.15 & 6.66 & 6.84 & 6.91 & 12.66 & 12.27 & 12.85 & 12.64 & 13.34 \\
\hline $\mathrm{Db} 15$ & 0.22 & 0.34 & 0.36 & 0.42 & 2.85 & 7.97 & 9.23 & 6.90 & 6.93 & 6.86 & 12.67 & 12.24 & 12.72 & 12.62 & 13.22 \\
\hline $\mathrm{Dbl6}$ & 0.22 & 0.34 & 0.39 & 0.42 & 2.86 & 8.00 & 9.20 & 6.73 & 6.83 & 6.85 & 12.75 & 12.27 & 12.77 & 12.53 & 13.28 \\
\hline $\mathrm{Dbl} 7$ & 0.27 & 0.34 & 0.39 & 0.42 & 2.82 & 7.93 & 9.26 & 6.69 & 6.83 & 6.93 & 12.60 & 12.23 & 12.78 & 12.64 & 13.23 \\
\hline Db18 & 0.27 & 0.34 & 0.36 & 0.42 & 2.86 & 7.93 & 9.22 & 6.87 & 6.90 & 6.87 & 12.65 & 12.23 & 12.70 & 12.53 & 13.24 \\
\hline Dbla & 0.22 & 0.34 & 0.39 & 0.42 & 2.87 & 8.05 & 9.26 & 6.78 & 6.86 & 6.98 & 12.63 & 12.24 & 12.71 & 12.66 & 13.31 \\
\hline $\mathrm{Db} 20$ & 0.27 & 0.34 & 0.39 & 0.42 & 2.84 & 7.84 & 9.10 & 6.63 & 6.84 & 6.91 & 12.59 & 12.21 & 12.76 & 12.44 & 13.14 \\
\hline Syml & 0.20 & 0.29 & 0.32 & 0.37 & 2.16 & 8.24 & 9.47 & 7.25 & 7.30 & 6.88 & 12.98 & 12.22 & 15.04 & 15.37 & 14.89 \\
\hline Sym2 & 0.21 & 0.33 & 0.35 & 0.38 & 2.45 & 8.30 & 9.53 & 6.98 & 6.94 & 6.88 & 12.57 & 12.24 & 13.33 & 13.77 & 13.58 \\
\hline $\operatorname{Sym} 3$ & 0.22 & 0.30 & 0.36 & 0.42 & 2.62 & 7.97 & 9.35 & 6.79 & 6.84 & 6.92 & 12.85 & 12.30 & 13.09 & 13.17 & 13.44 \\
\hline Sym 4 & 0.22 & 0.33 & 0.36 & 0.42 & 2.69 & 7.95 & 9.26 & 6.81 & 6.86 & 6.89 & 12.83 & 12.29 & 13.03 & 12.97 & 13.28 \\
\hline Sym5 & 0.22 & 0.35 & 0.36 & 0.40 & 2.73 & 7.99 & 9.48 & 6.80 & 6.86 & 6.77 & 12.72 & 12.21 & 13.03 & 12.87 & 13.13 \\
\hline Sym6 & 0.22 & 0.34 & 0.36 & 0.42 & 2.80 & 7.76 & 9.18 & 6.82 & 6.88 & 6.85 & 12.72 & 12.32 & 13.02 & 12.97 & 13.31 \\
\hline Sym7 & 0.22 & 0.34 & 0.36 & 0.42 & 2.80 & 8.05 & 9.34 & 6.73 & 6.81 & 6.94 & 12.70 & 12.27 & 12.85 & 12.70 & 13.08 \\
\hline Sym 8 & 0.21 & 0.34 & 0.36 & 0.42 & 2.82 & 7.81 & 9.23 & 6.84 & 6.95 & 6.85 & 12.73 & 12.30 & 12.99 & 12.74 & 13.27 \\
\hline Sym9 & 0.22 & 0.34 & 0.38 & 0.42 & 2.82 & 7.81 & 9.16 & 6.61 & 6.82 & 6.93 & 12.61 & 12.27 & 12.94 & 12.95 & 13.35 \\
\hline Sym10 & 0.22 & 0.34 & 0.36 & 0.42 & 2.90 & 7.92 & 9.33 & 6.85 & 6.90 & 6.93 & 12.69 & 12.31 & 12.79 & 12.66 & 13.16 \\
\hline Syml1 & 0.22 & 0.34 & 0.39 & 0.42 & 2.82 & 7.76 & 9.17 & 6.65 & 6.84 & 6.90 & 12.75 & 12.28 & 12.88 & 12.67 & 13.31 \\
\hline Sym12 & 0.22 & 0.35 & 0.36 & 0.42 & 2.80 & 8.04 & 9.21 & 6.75 & 6.87 & 6.84 & 12.74 & 12.32 & 12.81 & 12.64 & 13.22 \\
\hline Sym13 & 0.27 & 0.34 & 0.38 & 0.42 & 2.82 & 7.84 & 9.32 & 6.77 & 6.90 & 6.90 & 12.61 & 12.26 & 12.82 & 12.74 & 13.23 \\
\hline Sym14 & 0.22 & 0.34 & 0.39 & 0.42 & 2.75 & 8.00 & 9.20 & 6.77 & 6.87 & 6.91 & 12.61 & 12.29 & 12.89 & 12.67 & 13.39 \\
\hline Sym15 & 0.27 & 0.34 & 0.38 & 0.42 & 2.86 & 7.77 & 9.22 & 6.81 & 6.91 & 6.95 & 12.80 & 12.25 & 12.63 & 12.49 & 13.11 \\
\hline Syml6 & 0.22 & 0.34 & 0.39 & 0.42 & 2.77 & 7.96 & 9.18 & 6.83 & 6.82 & 6.90 & 12.72 & 12.27 & 12.82 & 12.52 & 13.26 \\
\hline Sym17 & 0.22 & 0.34 & 0.38 & 0.42 & 2.90 & 7.78 & 9.22 & 6.83 & 6.88 & 6.81 & 12.63 & 12.24 & 12.84 & 12.55 & 13.32 \\
\hline Sym18 & 0.27 & 0.34 & 0.38 & 0.42 & 2.82 & 7.95 & 9.26 & 6.73 & 6.88 & 6.90 & 12.61 & 12.25 & 12.78 & 12.50 & 13.18 \\
\hline Sym19 & 0.22 & 0.34 & 0.38 & 0.42 & 2.92 & 7.94 & 9.15 & 6.83 & 6.96 & 6.87 & 12.65 & 12.21 & 12.77 & 12.61 & 13.32 \\
\hline $\operatorname{Sym} 20$ & 0.27 & 0.34 & 0.39 & 0.42 & 2.83 & 7.87 & 9.17 & 6.68 & 6.87 & 7.01 & 12.72 & 12.21 & 12.72 & 12.40 & 13.13 \\
\hline Coifl & 0.21 & 0.31 & 0.35 & 0.38 & 2.48 & 8.19 & 9.45 & 6.79 & 6.87 & 6.95 & 12.82 & 12.16 & 13.55 & 13.65 & 13.57 \\
\hline Coif2 & 0.22 & 0.31 & 0.36 & 0.42 & 2.74 & 7.95 & 9.32 & 6.89 & 6.99 & 6.86 & 12.79 & 12.30 & 13.07 & 13.08 & 13.33 \\
\hline Coif3 & 0.22 & 0.34 & 0.36 & 0.42 & 2.79 & 7.93 & 9.32 & 6.75 & 6.91 & 6.78 & 12.72 & 12.26 & 12.90 & 12.78 & 13.22 \\
\hline Coif4 & 0.22 & 0.34 & 0.39 & 0.42 & 2.74 & 7.90 & 9.26 & 6.81 & 6.86 & 6.82 & 12.71 & 12.31 & 12.95 & 12.76 & 13.25 \\
\hline Coifs & 0.22 & 0.34 & 0.38 & 0.42 & 2.82 & 7.95 & 9.20 & 6.66 & 6.80 & 6.93 & 12.71 & 12.27 & 12.74 & 12.61 & 13.20 \\
\hline
\end{tabular}


Signal \& Image Processing: An International Journal (SIPIJ) Vol.11, No.1, February 2020

Table 2: Comparison of the simulated frequency in Beta and Gamma rhythms for the 45 MWTs.

\begin{tabular}{|c|c|c|c|c|c|c|c|c|c|c|}
\hline \multirow{2}{*}{ MWT } & \multicolumn{5}{|c|}{ Simulated frequency in Beta rhvthm $(\mathrm{Hz})$} & \multicolumn{5}{|c|}{ Simulated frequency in Gammarhythm (Hz) } \\
\hline & SET A & SET B & SET C & SET D & SET E & SET A & SET B & SET C & SET D & SET E \\
\hline $\mathrm{Dbl}$ & 29.25 & 31.93 & 33.97 & 33.89 & 32.94 & 66.52 & 70.33 & 71.69 & 74.16 & 75.93 \\
\hline $\mathrm{Db} 2$ & 26.18 & 30.91 & 26.01 & 25.38 & 27.97 & 51.75 & 60.18 & 52.75 & 55.24 & 70.22 \\
\hline $\mathrm{Db} 3$ & 25.09 & 28.65 & 25.17 & 24.86 & 26.76 & 50.76 & 53.31 & 51.26 & 52.49 & 64.30 \\
\hline $\mathrm{Db} 4$ & 24.79 & 26.81 & 24.48 & 24.25 & 25.28 & 50.12 & 50.00 & 50.51 & 51.12 & 59.36 \\
\hline Db5 & 24.71 & 26.67 & 24.36 & 23.85 & 25.60 & 50.01 & 50.00 & 50.28 & 50.33 & 56.83 \\
\hline $\mathrm{Db} 6$ & 24.46 & 25.72 & 24.40 & 24.18 & 24.86 & 50.01 & 50.00 & 50.04 & 50.19 & 55.10 \\
\hline $\mathrm{Db} 7$ & 24.53 & 25.01 & 24.27 & 24.07 & 24.37 & 50.01 & 50.00 & 50.08 & 50.08 & 53.79 \\
\hline $\mathrm{Db} 8$ & 24.45 & 24.61 & 24.23 & 23.78 & 24.44 & 50.01 & 50.00 & 50.05 & 50.08 & 52.63 \\
\hline $\mathrm{Db} 9$ & 24.45 & 24.72 & 24.04 & 23.93 & 24.29 & 50.01 & 50.00 & 50.08 & 50.08 & 52.16 \\
\hline Dblo & 24.42 & 24.70 & 24.23 & 24.00 & 24.13 & 50.01 & 50.00 & 50.11 & 50.18 & 51.87 \\
\hline Dbl1 & 24.42 & 24.31 & 24.18 & 23.64 & 24.09 & 50.01 & 50.00 & 50.04 & 50.07 & 51.39 \\
\hline $\mathrm{Db} 12$ & 24.27 & 24.54 & 24.12 & 23.81 & 24.11 & 50.01 & 50.00 & 50.06 & 50.15 & 50.76 \\
\hline Dbl3 & 24.36 & 24.47 & 24.08 & 23.79 & 23.89 & 50.01 & 50.00 & 50.04 & 50.15 & 50.50 \\
\hline Dbl4 & 24.61 & 24.11 & 24.03 & 23.62 & 24.05 & 50.01 & 50.00 & 50.04 & 50.08 & 49.97 \\
\hline Dbls & 24.27 & 24.20 & 24.04 & 23.75 & 23.97 & 50.01 & 50.00 & 50.04 & 50.08 & 49.48 \\
\hline Dbl6 & 24.41 & 24.33 & 24.03 & 23.75 & 23.81 & 50.01 & 50.00 & 50.04 & 50.08 & 49.62 \\
\hline Dbi7 & 24.58 & 24.00 & 24.00 & 23.64 & 23.91 & 50.01 & 50.00 & 50.04 & 50.15 & 49.20 \\
\hline Db18 & 24.41 & 24.21 & 24.04 & 23.69 & 23.99 & 50.01 & 50.00 & 50.04 & 50.15 & 48.59 \\
\hline Db19 & 24.39 & 24.19 & 23.97 & 23.69 & 23.77 & 50.01 & 50.00 & 50.04 & 50.15 & 48.76 \\
\hline $\mathrm{Db} 20$ & 24.48 & 23.73 & 23.93 & 23.70 & 23.80 & 50.01 & 50.00 & 50.04 & 50.15 & 49.11 \\
\hline Syml & 29.25 & 31.93 & 33.97 & 33.89 & 32.94 & 66.52 & 70.33 & 71.69 & 74.16 & 75.93 \\
\hline Sym2 & 26.18 & 30.91 & 26.01 & 25.38 & 27.97 & 51.75 & 60.18 & 52.75 & 55.24 & 70.22 \\
\hline Sym 3 & 25.09 & 28.65 & 25.17 & 24.86 & 26.76 & 50.76 & 53.31 & 51.26 & 52.49 & 64.30 \\
\hline Sym 4 & 24.62 & 26.88 & 24.54 & 24.43 & 25.46 & 50.25 & 50.00 & 50.88 & 51.60 & 59.17 \\
\hline Syms & 24.91 & 26.16 & 24.51 & 24.03 & 24.79 & 50.09 & 50.00 & 50.28 & 50.08 & 56.45 \\
\hline Sym6 & 24.44 & 25.48 & 24.32 & 23.97 & 25.01 & 50.01 & 50.00 & 50.07 & 50.19 & 55.06 \\
\hline Sym 7 & 24.36 & 25.12 & 24.27 & 24.08 & 24.53 & 50.01 & 50.00 & 50.15 & 50.11 & 53.68 \\
\hline Sym 8 & 24.57 & 24.86 & 24.30 & 24.03 & 24.25 & 50.01 & 50.00 & 50.04 & 50.16 & 52.65 \\
\hline Symg & 24.71 & 24.50 & 24.27 & 23.78 & 24.11 & 50.00 & 50.00 & 50.05 & 50.08 & 51.98 \\
\hline Sym 10 & 24.38 & 25.08 & 24.15 & 23.77 & 24.25 & 50.01 & 50.00 & 50.04 & 50.15 & 51.51 \\
\hline Syml1 & 24.20 & 24.60 & 24.07 & 23.84 & 24.09 & 50.01 & 50.00 & 50.04 & 50.07 & 51.35 \\
\hline Sym 12 & 24.39 & 24.27 & 24.16 & 23.90 & 23.98 & 50.01 & 50.00 & 50.08 & 50.08 & 50.70 \\
\hline Sym 13 & 24.58 & 24.17 & 24.08 & 23.77 & 24.04 & 50.01 & 50.00 & 50.04 & 50.08 & 50.05 \\
\hline Sym 14 & 24.44 & 24.37 & 24.04 & 23.59 & 24.19 & 50.01 & 50.00 & 50.04 & 50.08 & 50.33 \\
\hline Sym 15 & 24.19 & 24.39 & 23.97 & 23.72 & 24.00 & 50.01 & 50.00 & 50.04 & 50.15 & 49.86 \\
\hline Sym 16 & 24.35 & 24.31 & 23.99 & 23.77 & 23.89 & 50.01 & 50.00 & 50.04 & 50.08 & 49.69 \\
\hline Sym 17 & 24.46 & 24.06 & 24.00 & 23.60 & 23.91 & 50.01 & 50.00 & 50.04 & 50.08 & 49.10 \\
\hline Symls & 24.27 & 23.94 & 24.03 & 23.66 & 23.95 & 50.01 & & 50.04 & 50.15 & 49.41 \\
\hline Sym19 & 24.18 & 23.87 & 23.98 & 23.66 & 23.84 & 50.01 & 50.00 & 50.04 & 50.15 & 49.09 \\
\hline Sym20 & 24.40 & 24.03 & 23.95 & 23.67 & 23.76 & 50.01 & 50.00 & 50.04 & 50.15 & 49.16 \\
\hline Coifl & 26.60 & 30.48 & 25.38 & 26.50 & 27.75 & 52.08 & 59.57 & 52.43 & 54.81 & 70.07 \\
\hline Coif2 & 24.72 & 26.67 & 24.51 & 24.32 & 25.13 & 50.17 & 50.00 & 50.96 & 51.20 & 58.57 \\
\hline Coif & 24.47 & 25.17 & 24.40 & 24.07 & 24.30 & 50.01 & 50.00 & 50.04 & 50.19 & 54.36 \\
\hline Coift & 24.62 & 24.82 & 24.32 & 24.02 & 24.15 & 50.01 & 50.00 & 50.04 & 50.15 & 52.37 \\
\hline Coifs & 24.63 & 24.51 & 24.18 & 23.85 & 24.02 & 50.01 & 50.00 & 50.04 & 50.15 & 51.26 \\
\hline
\end{tabular}


Signal \& Image Processing: An International Journal (SIPIJ) Vol.11, No.1, February 2020

Table 3: Comparison of the PRD and SNR for the 45 MWTs.

\begin{tabular}{|c|c|c|c|c|c|c|c|c|c|c|}
\hline \multirow{2}{*}{ MWT } & \multicolumn{5}{|c|}{ PRD ( $(96)$} & \multicolumn{5}{|c|}{ SNR (Db) } \\
\hline & SET A & SET B & SET C & SET D & SET E & SET A & SET B & SET C & SET D & SET E \\
\hline $\mathrm{Dbl}$ & $6.430^{*} 10^{-14}$ & $5.986^{4} 10^{-14}$ & $5.507^{4} 10^{-14}$ & $6.324 * 10^{-16}$ & $5.235^{*} 10^{-14}$ & 606.623 & 609.650 & 607.982 & 608.191 & 611.650 \\
\hline $\mathrm{Db} 2$ & $1.408 * 10^{-10}$ & $1.653 * 10^{-10}$ & $1.094 * 10^{-10}$ & $1.183 * 10^{-10}$ & $1.626^{*}+10^{-10}$ & 453.749 & 450.515 & 458.931 & 457.503 & 451.104 \\
\hline $\mathrm{Db} 3$ & $1.539 * 10^{-6}$ & $1.688 * 10^{-9}$ & $1.449 * 10^{-8}$ & $1.528 * 10^{-6}$ & $1.791 * 10^{-8}$ & 405.894 & 404.040 & 407.137 & 406.153 & 402.916 \\
\hline $\mathrm{Db} 4$ & $2.890 * 10^{-10}$ & $3.239 * 10^{-10}$ & $2.676^{*} 10^{-10}$ & $2.837^{*}+10^{-10}$ & $3.36710^{-10}$ & 439.337 & 437.045 & 440.925 & 439.837 & 436.353 \\
\hline Db5 & $4.243 * 10^{-10}$ & $4.672 * 10^{-10}$ & $4.096^{*} 10^{-10}$ & $4.312 * 10^{-10}$ & $4.984 * 10^{-10}$ & 431.653 & 429.724 & 432.387 & 431.410 & 428.498 \\
\hline $\mathrm{Db} 6$ & $2.396^{*} 10^{-10}$ & $2.777^{*} 10^{-10}$ & $2.325 * 10^{-10}$ & $2.478 * 10^{-10}$ & $2.652 * 10^{-10}$ & 443.089 & 440.320 & 443.693 & 442.497 & 441.093 \\
\hline $\mathrm{Db} 7$ & $3.457 * 10^{-10}$ & $3.981 * 10^{-10}$ & $2.971 * 10^{-10}$ & $3.190 * 10^{-10}$ & $4.052 * 10^{-10}$ & 435.767 & 432.930 & 438.880 & 437.552 & 432.747 \\
\hline $\mathrm{Db8}$ & $7.076^{4} 10^{-10}$ & $7.704 * 10^{-10}$ & $7.716^{4} 10^{-10}$ & $7.141 * 10^{-10}$ & $8.312 * 10^{-10}$ & 421.434 & 419.762 & 422.512 & 421.355 & 418.274 \\
\hline $\mathrm{Db} 9$ & $7.353 * 10^{-9}$ & $9.016^{*} 10^{-8}$ & $5.379 * 10^{-8}$ & $5.858 * 10^{-6}$ & $8.381 * 10^{-6}$ & 374.657 & 370.632 & 381.083 & 379.538 & 372.369 \\
\hline $\mathrm{Db} 10$ & $8.799 * 10^{-10}$ & $1.077 * 10^{-8}$ & $6.954 * 10^{-10}$ & $7.523 * 10^{-10}$ & $1.005 * 10^{-9}$ & 417.093 & 413.100 & 421.911 & 420.475 & 414.649 \\
\hline Dbll & $9.955^{*}+10^{-12}$ & $1.114^{*} 10^{-11}$ & $9.664 * 10^{-12}$ & $1.018 * 10^{-11}$ & $1.164 * 10^{-11}$ & 506.697 & 504.461 & 507.317 & 506.340 & 503.626 \\
\hline $\mathrm{Db} 12$ & $2.290 * 10^{-11}$ & $2.570^{*} 10^{-11}$ & $2.183 * 10^{-11}$ & $2.305 * 10^{-11}$ & $2.681 * 10^{-11}$ & 490.037 & 487.733 & 491.026 & 490.000 & 486.962 \\
\hline $\mathrm{Db} 13$ & $1.488 * 10^{-12}$ & $1.523 * 10^{-12}$ & $1.684^{*} 10^{-12}$ & $1.705 * 10^{-12}$ & $1.687 * 10^{-12}$ & 544.712 & 544.279 & 542.214 & 541.98 & 542.283 \\
\hline $\mathrm{Dbl4}$ & $2.255 * 10^{-10}$ & $2.471 * 10^{-10}$ & $2.267 * 10^{-10}$ & $2.368 * 10^{-10}$ & $2.642 * 10^{-10}$ & 444.286 & 442.470 & 444.195 & 443.367 & 441.177 \\
\hline Dbl5 & $4.002 * 10^{-11}$ & $4.504 * 10^{-11}$ & $3.853 * 10^{-11}$ & $4.062 * 10^{-11}$ & $4.653 * 10^{-11}$ & 478.873 & 476.536 & 479.654 & 478.663 & 475.932 \\
\hline $\mathrm{Dbl} 6$ & $1.038 * 10^{-10}$ & $1.137 * 10^{-10}$ & $1.049 * 10^{-10}$ & $1.084 * 10^{-10}$ & $1.227 * 10^{-10}$ & 459.803 & 458.977 & 459.615 & 458.973 & 456.545 \\
\hline $\mathrm{Db} 17$ & $1.453 * 10^{-8}$ & $1.538 * 10^{-8}$ & $1.530^{*} 10^{-8}$ & $1.580^{*} 10^{-8}$ & $1.693 * 10^{-8}$ & 407.024 & 405.889 & 405.989 & 405.373 & 404.017 \\
\hline Dbl8 & $1.581 * 10^{-9}$ & $1.677 * 10^{-8}$ & $1.667 * 10^{-8}$ & $1.725 * 10^{-8}$ & $1.835 * 10^{-8}$ & 405.331 & 404.181 & 404.274 & 403.624 & 402.409 \\
\hline $\mathrm{Db} 19$ & $4.420^{*} 10^{-10}$ & $4.771 * 10^{-10}$ & $4.571 * 10^{-10}$ & $4.713 * 10^{-10}$ & $5.181 * 10^{-10}$ & 430.822 & 429.304 & 430.160 & 429.571 & 427.742 \\
\hline $\mathrm{Db} 20$ & $1.895 * 10^{-9}$ & $1.984 * 10^{-9}$ & $2.029 * 10^{-8}$ & $2.098 \times 10^{-9}$ & $2.173 * 10^{-9}$ & 401.715 & 400.844 & 400.340 & 399.707 & 399.021 \\
\hline Syml & $6.430^{*} 10^{-14}$ & $5.986^{*} 10^{-14}$ & $6.507 * 10^{-14}$ & $6.324 * 10^{-14}$ & $5.235^{*} 10^{-14}$ & 608.623 & 609.650 & 607.982 & 608.191 & 611.650 \\
\hline Sym 2 & $1.408^{*} 10^{-10}$ & $1.653 * 10^{-10}$ & $1.094^{*} 10^{-10}$ & $1.183 * 10^{-10}$ & $1.626^{*} 10^{-10}$ & 453.749 & 450.515 & 458.931 & 457.503 & 451.104 \\
\hline Sym3 & $1.539 * 10^{-8}$ & $1.688 * 10^{-8}$ & $1.449 * 10^{-8}$ & $1.528 * 10^{-8}$ & $1.791 * 10^{-8}$ & 405.894 & 404.040 & 407.137 & 406.153 & 402.916 \\
\hline Sym4 & $1.166^{*}+10^{-10}$ & $1.390 * 10^{-10}$ & $8.479 * 10^{-11}$ & $9.206^{*} 10^{-11}$ & $1.332 * 10^{-10}$ & 457.537 & 454.006 & 464.097 & 462.585 & 455.187 \\
\hline Sym 5 & $4.031 * 10^{-11}$ & $4.819 * 10^{-11}$ & $2.887^{4} 10^{-11}$ & $3.139 * 10^{-11}$ & $4.584 * 10^{-11}$ & 478.782 & 475.193 & 485.654 & 484.117 & 476.523 \\
\hline Sym6 & $1.853 * 10^{-10}$ & $2.226^{*} 10^{-10}$ & $1.359 * 10^{-10}$ & $1.478 * 10^{-10}$ & $2.121 * 10^{-10}$ & 448.266 & 444.596 & 454.658 & 453.115 & 445.876 \\
\hline Sym 7 & $1.668^{*}+10^{-10}$ & $2.058 * 10^{-10}$ & $1.159 * 10^{-10}$ & $1.262 * 10^{-10}$ & $1.865^{*}+10^{-10}$ & 450.396 & 446.263 & 457.857 & 456.324 & 448.501 \\
\hline Sym 8 & $3.755^{*} 10^{-11}$ & $3.727 * 10^{-11}$ & $3.942 * 10^{-11}$ & $4.092 * 10^{-11}$ & $4.278 * 10^{-11}$ & 480.177 & 480.621 & 479.202 & 478.580 & 477.648 \\
\hline Sym9 & $9.083 * 10^{-12}$ & $1.058^{*} 10^{-12}$ & $6.676^{*} 10^{-12}$ & $7.226^{*} 10^{-12}$ & $1.040^{*} 10^{-12}$ & 554.632 & 551.548 & 560.975 & 559.523 & 552.214 \\
\hline Sym10 & $1.052^{*} 10^{-12}$ & $1.235^{*} 10^{-12}$ & $8.104^{*} 10^{-12}$ & $8.713 * 10^{-12}$ & $1.202 * 10^{-12}$ & 551.701 & 548.463 & 557.061 & 555.726 & 549.288 \\
\hline Syml1 & $1.046^{*} 10^{-12}$ & $1.104^{*} 10^{-12}$ & $8.501 * 10^{-13}$ & $8.666^{*} 10^{-12}$ & $1.081 * 10^{-12}$ & 551.830 & 550.801 & 555.923 & 555.541 & 551.213 \\
\hline Sym12 & $1.394 * 10^{-11}$ & $1.605^{4} 10^{-11}$ & $1.174 * 10^{-11}$ & $1.206^{*} 10^{-11}$ & $1.624^{*}+10^{-11}$ & 499.994 & 497.151 & 503.514 & 502.213 & 497.095 \\
\hline Sym13 & $1.337 * 10^{-11}$ & $1.554 * 10^{-11}$ & $1.115^{*} 10^{-11}$ & $1.199 * 10^{-11}$ & $1.560 * 10^{-11}$ & 500.829 & 497.790 & 504.546 & 503.217 & 497.929 \\
\hline Syml4 & $8.094 * 10^{-11}$ & $9.379 * 10^{-11}$ & $6.673 * 10^{-11}$ & $7.182 * 10^{-11}$ & $9.420 * 10^{-11}$ & 464.811 & 461.839 & 468.778 & 467.427 & 461.967 \\
\hline Syml5 & $2.249 * 10^{-11}$ & $2.593 * 10^{-11}$ & $1.853 * 10^{-11}$ & $1.991 * 10^{-11}$ & $2.623 * 10^{-11}$ & 490.422 & 487.5032 & 494.409 & 493.087 & 487.556 \\
\hline Syml6 & $2.185 * 10^{-10}$ & $2.595 * 10^{-10}$ & $1.643 * 10^{-10}$ & $1.784 * 10^{-10}$ & $2.514 * 10^{-10}$ & 444.963 & 441.509 & 450.833 & 449.332 & 442.445 \\
\hline Sym17 & $5.115^{*} 10^{-11}$ & $5.857^{*} 10^{-11}$ & $3.658^{*} 10^{-11}$ & $3.938 * 10^{-11}$ & $5.674 * 10^{-11}$ & 474.022 & 471.304 & 480.866 & 479.531 & 472.175 \\
\hline Sym18 & $2.514 * 10^{-8}$ & $2.976^{4} 10^{-8}$ & $1.919 * 10^{-8}$ & $2.081 * 10^{-9}$ & $2.899 * 10^{-8}$ & 396.104 & 392.709 & 401.662 & 400.181 & 393.524 \\
\hline Sym19 & $1.466^{*}+10^{-10}$ & $1.703 * 10^{-10}$ & $1.120 * 10^{-10}$ & $1.214 * 10^{-10}$ & $1.681 * 10^{-10}$ & 452.943 & 449.917 & 458.479 & 457.026 & 450.441 \\
\hline Sym20 & $8.968 * 10^{-8}$ & $1.050^{*} 10^{-4}$ & $7.133 * 10^{-8}$ & $7.704 * 10^{-8}$ & $1.040^{*} 10^{-2}$ & 370.664 & 367.478 & 375.373 & 373.961 & 367.921 \\
\hline Coifl & $2.143 * 10^{-10}$ & $2.547 * 10^{-10}$ & $1.572 * 10^{-10}$ & $1.705 * 10^{-10}$ & $2.449 * 10^{-10}$ & 445.366 & 441.884 & 451.743 & 450.255 & 442.982 \\
\hline Coif2 & $2.556^{4}+10^{-8}$ & $2.936^{4} 10^{-8}$ & $2.138+10^{-8}$ & $2.293 * 10^{-9}$ & $2.978 * 10^{-9}$ & 395.756 & 392.962 & 399.424 & 398.140 & 392.877 \\
\hline Coifs & $1.261 * 10^{-10}$ & $1.463 * 10^{-10}$ & $1.002 * 10^{-10}$ & $1.078 * 10^{-10}$ & $1.421 * 10^{-10}$ & 455.976 & 453.037 & 460.675 & 459.354 & 453.776 \\
\hline Coift & $5.902 * 10^{-9}$ & $7.103 * 10^{-9}$ & $4.365 * 10^{-8}$ & $4.685 * 10^{-9}$ & $6.669 * 10^{-9}$ & 379.076 & 375.352 & 385.284 & 383.990 & 376.856 \\
\hline Coifs & $1.316^{*} 10^{-6}$ & $1.618^{*}+10^{-6}$ & $9.386^{4} 10^{-7}$ & $1.014^{*} 10^{-6}$ & $1.489 * 10^{-6}$ & 270.958 & 266.879 & 277.898 & 276.475 & 268.792 \\
\hline
\end{tabular}

Table 1 and Table 2 rely on the comparison between MWTs in terms of simulated frequency. These simulated frequencies represent the mean of each SET of EEG signals for different MWT. In Delta rhythm, Db8 presents highest values of simulated frequencies of $0.22 \mathrm{~Hz}, 0.35 \mathrm{~Hz}, 0.39$ $\mathrm{Hz}, 0.42 \mathrm{~Hz}$ and $2.82 \mathrm{~Hz}$ for SET A, SET B, SET C, SETD and SET E, respectively. The results showed thatDb1 and Sym1 present equal and highest simulated frequencies in Theta, Alpha, Beta and Gamma rhythms.Simulated frequencies in Theta rhythm are $8.24 \mathrm{~Hz}, 9.47 \mathrm{~Hz}, 7.25 \mathrm{~Hz}, 7.30$ $\mathrm{Hz}$ and $6.86 \mathrm{~Hz}$ for SETs A, B, C, D and E, respectively. Also, highest values in Alpha rhythm are $12.98 \mathrm{~Hz}, 12.22 \mathrm{~Hz}, 15.04 \mathrm{~Hz}, 15.37 \mathrm{~Hz}$ and $14.89 \mathrm{~Hz}$ for SETs A, B, C, D and E, respectively. Highest values of $29.25 \mathrm{~Hz}, 31.93 \mathrm{~Hz}, 33.97 \mathrm{~Hz}, 33.89 \mathrm{~Hz}$ and $32.94 \mathrm{~Hz}$ for SETs A, B, C, D and E, respectively, in Beta rhythm. Gamma rhythm also gives highest values of 66.52 $\mathrm{Hz}, 70.33 \mathrm{~Hz}, 71.69 \mathrm{~Hz}, 74.16 \mathrm{~Hz}$ and $75.93 \mathrm{~Hz}$. These simulated frequencies are high, but they are not correlated to the rhythms defined by the literature. In regards of this, it is possible to state that $\mathrm{Db} 1$ and Sym1 are not suitable for the decomposition of EEG signals in their different rhythms. Then, knowing that decomposed rhythms must be more correlated to the ones defined by the literature, Table 1 and Table 2 demonstrated that Db4 presents more correlated simulated frequencies and can be suitable as MWT for EEG rhythms decomposition. 
Signal \& Image Processing: An International Journal (SIPIJ) Vol.11, No.1, February 2020

Table 2 presents the PRD and SNR obtained after reconstruction of the EEG signals. It is related that $\mathrm{Db} 1$ and Sym1 give best performance. In terms of PRD, Db1 and Sym1 give smallest PRD of $6.430 * 10^{-14} \%, 5.986^{*} 10^{-14} \%, 5.507 * 10^{-14} \%, 6.324 * 10^{-14} \%$ and $5.235^{*} 10^{-14} \%$ for SETs A, B, C, $\mathrm{D}$ and E, respectively. Also, these two MWT obtained highest SNR of $606.623 \mathrm{Db}, 609.650 \mathrm{Db}$, 607.982 Db, 608.191 Db and 611.650 Db for SETs A, B, C, D and E, respectively. These values of PRD and SNR permit us to postulate that some information is missing after reconstruction. Also, this demonstrates that Db1 and Sym1 are suitable as MWT for the reconstruction of the analyzed EEG signals.

Then, taking into account that the decomposition of EEG signals in their different rhythms is suitable when the reconstructed signal presents lower loss of information, and the obtained rhythms are more correlated to the ones defines by the literature, obtained results demonstrate that the signals are within the Db4 MWT. An example of EEG frequency bands decomposition using the suitable Db4 MWT is presented in Figure 3.

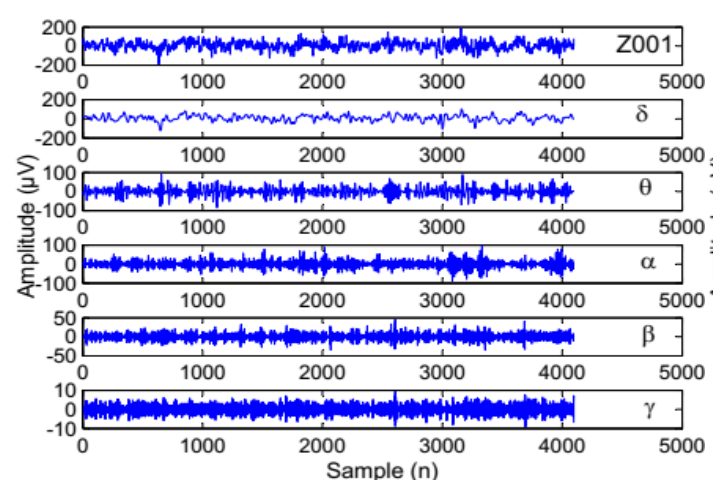

(i)

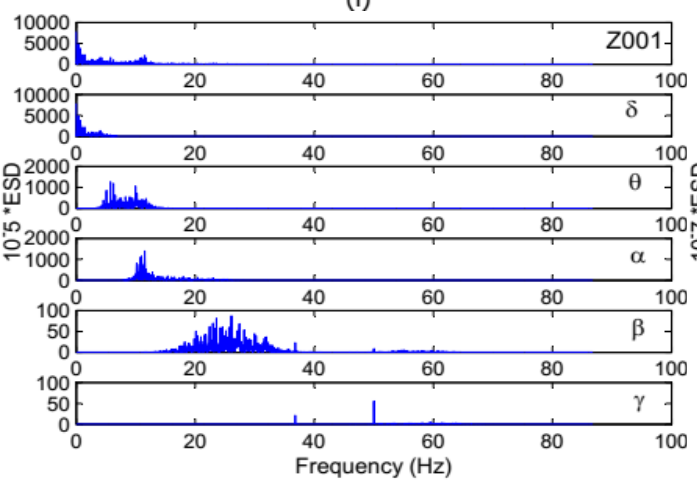

(ii)

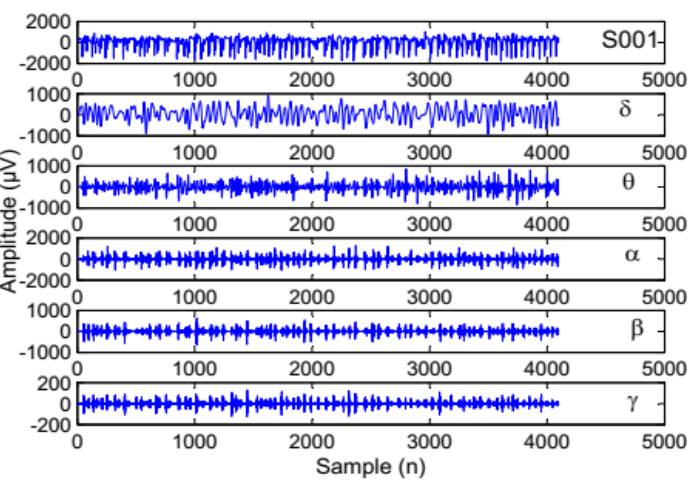

(i)

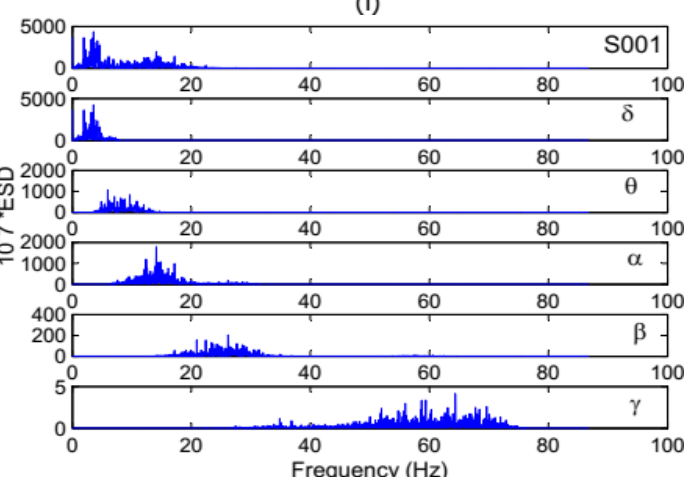

(ii)

(a)(b)

Figure 3: Frequency bands decomposition of a (a) normal EEG Z001 and a (b) seizure EEG S001 using WT with Db4 MWT.

\subsection{Features extraction and selection using LDA}

After frequency bands decomposition using the Db4 as suitable MWT, in total we have 26 features in the wavelet-based features set. This 26-dimension features vector derived using suitable MWT represents the amount of changes of the signal in frequency distribution. The statistical analysis of the extracted features is presented in Figure 4. According to the supervision of Figure 4, it is related that statistics, energy and entropy features extracted are representative for the normal, non-seizure and seizure EEG signals but still presents redundancy. Therefore, a selection process of discriminative features is necessary. 
Signal \& Image Processing: An International Journal (SIPIJ) Vol.11, No.1, February 2020
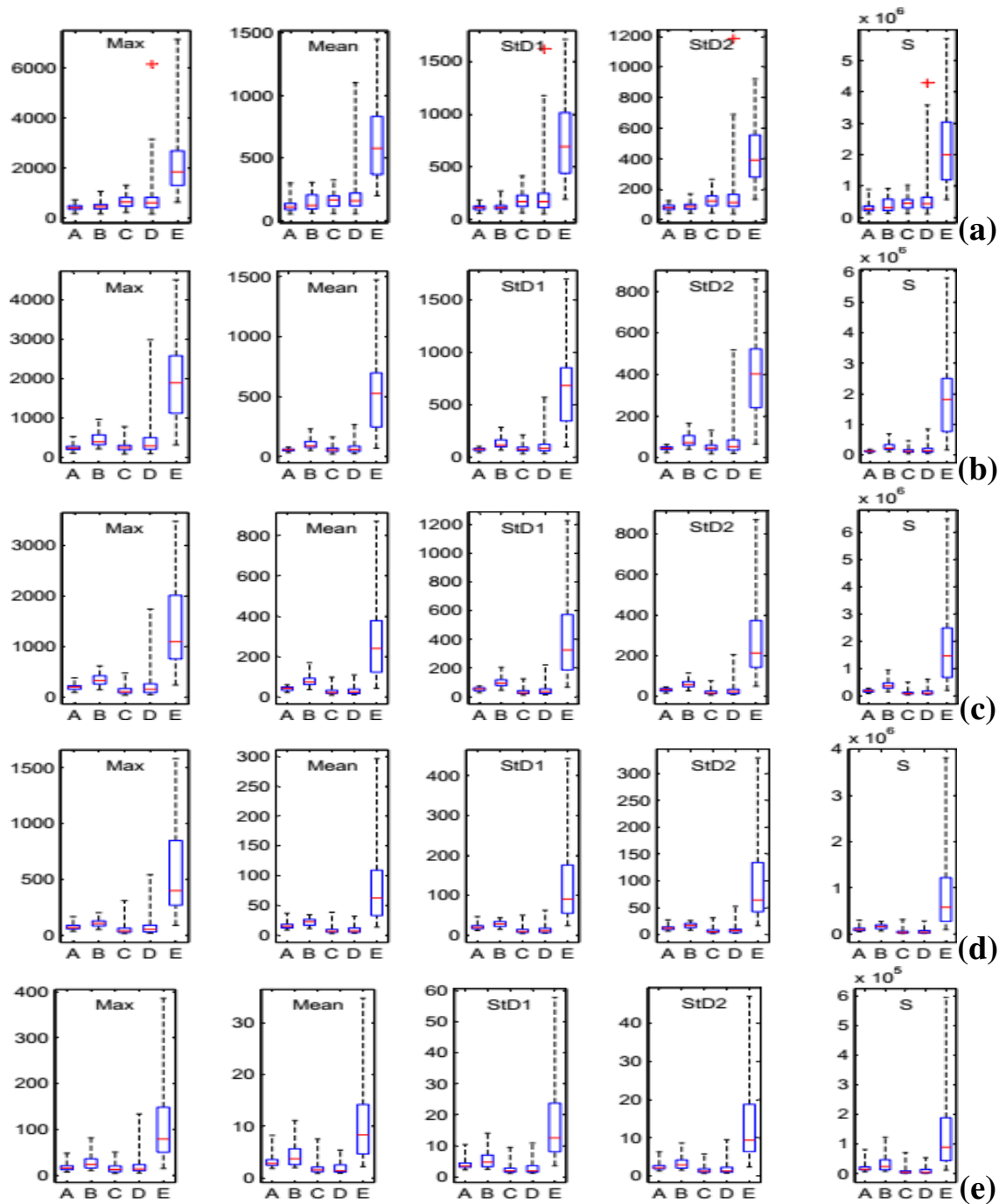

Figure 3: Comparison of statistics, entropy and energy features corresponding to the (a) Delta, (b) Theta, (c) Alpha, (d) Beta and (e) Gamma frequency bands using WT with Db4 MWT.

LDA is applied to select suitable, easily viewed and interpreted2-dimension features. For different discrimination problem, the first $40 \%$ of each SET are used to determine the useful linear discriminants for the feature's selection process. Figure 4 presents scatter plot of this selected LDA features. It is related that, this 2-dimension features vector represents the amount of changes in linear discriminant distribution, and carry most discriminative and representative information about their classes for twelve discrimination problems. 
Signal \& Image Processing: An International Journal (SIPIJ) Vol.11, No.1, February 2020
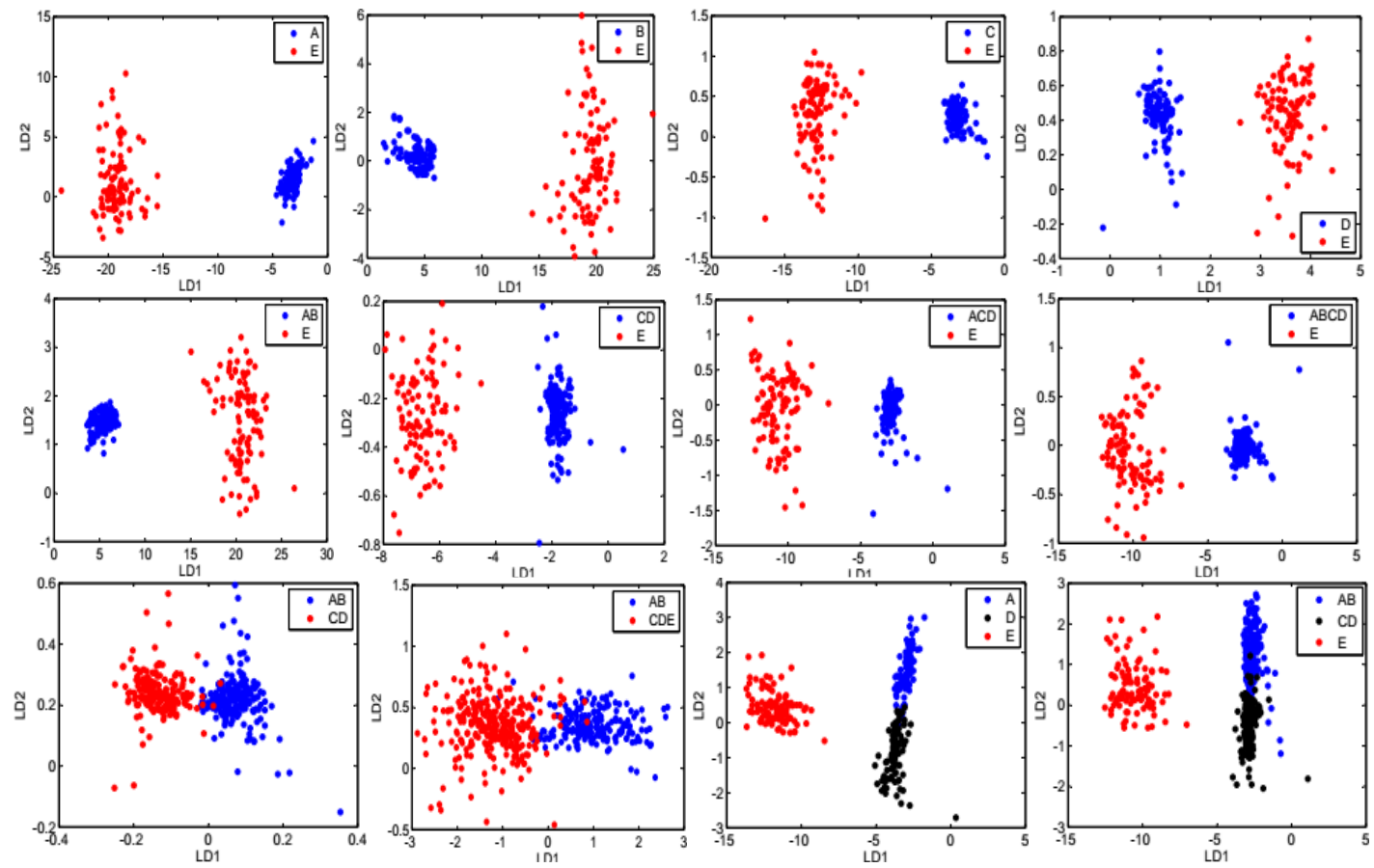

Figure 4: Scatter plot of selected LDA features for twelve discriminations problems.

\subsection{Discussion}

Although many works exploiting WT are presented for frequency bands decomposition, features extraction and classification of EEGs signals, the problems of MWT selection and discriminative features extraction still must be discussed.

In this study, in order to select the suitable MWT for four level wavelet decomposition, 45 MWTs are used. According to the frequency occupation of the decomposed bands, the PRD and the SNR, it is shown that Db4 is the suitable MWT even though it does not present the lowest PRDand the highest SNR as shown by Table 1 and Table 2. But, Db4 gathers the quasi-totality of information of the original signal on its wavelet coefficients and defines as much as possible the frequency bands to the ones defined in the literature. This MWT can also enable us to reduce overlapping that exist between decomposed frequency bands.

After the suitable MWT selection for frequency bands decomposition, the purpose of features extraction is generally applied. Firstly, some statistics, energy and entropy features are extracted directly from the frequency bands coefficients and form the original feature sets. Since these original feature sets are in high dimension and are irrelevant or redundant, as shown by Figure 3, it is a trade-off to select an optimal feature subset from the original feature set, and some kinds of feature dimensionality reduction, such as the well-known LDA, are employed to improve the discriminationperformance. The LDA algorithm is implemented to obtain a relatively low dimensional, but significantly informative and discriminative feature set. The result of the LDA features selection is shown in Figure 4. Clearly, each original feature space is mapped into a reduced two-dimensional feature space by using the LDA method.Compared to other works, the selected LDA feature vector is extremely discriminative and can be used as a benchmark of classification research using simple kernel machines in opposition tocomplex kernel machines. 
Signal \& Image Processing: An International Journal (SIPIJ) Vol.11, No.1, February 2020

\section{Conclusion And Prospectives}

The aim of this paper was to use WT with suitable MWT for efficient EEG frequency bands decomposition and features extraction. This study establishes that Db4wavelet is suitable for EEG frequency bands decomposition. Moreover, extracted and selected features related to wavelet coefficients with suitable MWT present a good discrimination for normal, non-seizure and seizureEEG signals. As prospects, these 2-dimension features can be used as a benchmark of classification research in future worksfor improvingclassification accuracies.

\section{REFERENCES}

[1] Lopes Da Silva F. I. H., Gonzales S. I. (2009) “Electroencephalography (EEG)”, Encyclopedia of Neurosciences, Academic Press, pp. 849-855.

[2] Song Y., Zhang J. (2013) “Automatic recognition of epileptic EEG patterns via extreme learning machine and multiresolution features extraction', Expert Systems and Applications, Vol. 40, No. 14, pp. 5477-5489, October.

[3] Pushpendra Singh, S. D. Joshi, R. K. Patney, Kaushik Saha (2016), "Fourier-based feature extraction for classification of EEG signals using EEG rhythms", Circuits Syst Signal Process, Vol. 35, pp. 3700-3715. DOI: 10.1007/s00034-015-0225-z.

[4] Ji Zhong, Qin Shuren, Peng Chenglin (2008) "Study on separation for the frequency bands of EEG signal and frequency band relative intensity analysis based upon EMD', Proceedings of the 7th WSEAS International Conference on Signal Processing, Robotics and Automation (ISPRA 08), ISSN: 1790-5117 151, University of Cambridge, UK, February 20-22.

[5] Wu Z., Huang N. E. (2009) "Ensemble empirical mode decomposition: a noise-assisted data analysis method", Adv. Adapt. Data Anal., Vol. 1, No. 1, pp. 1-41.

[6] Pachori R. B. and Bajaj V. (2011) "Analysis of normal and epileptic seizure eeg signals using empirical mode decomposition', Comput. Methods Progr. Biomed., Vol. 104, pp. 373-381.

[7] Pradip Sircar, Ram Bilas Pachori (2009) "Analysis of rhythms of EEG signals using orthogonal polynomial approximation', Conference Paper, January. DOI: 10.1145/1644993.1645025.

[8] Adeli H., Zhou Z., Dadmehr N. (2003) “Analysis of EEG records in an epileptic patient using wavelet transform”, Journal of Neurosciences Methods, Vol. 123, No. 1, pp. 69-87.

[9] Subasi Abdulhamit (2007) "Application of adaptative neuro-fussy inference system for epileptic seizure detection using wavelet features extraction', Computer in Biology and Medicine, Vol. 37 , No. 2, pp. 227-244.

[10] Subasi Abdulhamit (2007) "EEG signal classification using wavelet features extraction and a mixture of expert model', Elsevier Transactions on Expert System with Applications, Vol. 32, No. 5, pp. 1084-1093.

[11] Wu Ting, Yan Guo-Zheng, Yang Bang-Hua, Sun Hong (2008) 'EEG features extraction based on wavelet packet decomposition for brain computer interface", ELSEVIER Transactions on Measurement, Vol. 41, pp. 618-625.

[12] Saching Garg, Rakesh Narvey (2013) “Denoising and features extraction of eeg signal using wavelet transform', International Journal of Engineering Science and Technology (IJEST), Vol. 5, No. 6, pp. 1249-1253, June. ISSN: 0975-5462.

[13] Rosenblatt Mariel and al. (2014) "A quantitative analysis of an eeg epileptic record based on multiresolution wavelet coefficients", Entropy, Vol. 16, pp. 5976-6005. DOI: 10.3390/e16115976.

[14] Mohan Kumar C. E., Dharani Kumar S. V. (2014) "Wavelet based features extraction scheme of electroencephalogram,', International Journal of Innovative Research in Science, Engineering and Technology (IJIRSET), Vol. 3, Special Issue 1, February.

[15] Sachin M. Rathod and Kulkarni S. C. (2014) "classification of electroencephalogram using wavelet transform and neural network", International Journal of Science and Research (IJSR), Vol. 3, Issue 6, June.

[16] Alexandros T. Tzallas (2009) "Epileptic seizure detection in EEGs using time-frequency analysis", IEEE Transactions on Information Technology in Biomedicine, Vol. 13, No. 5, September. 
Signal \& Image Processing: An International Journal (SIPIJ) Vol.11, No.1, February 2020

[17] Noor K. Al-Kazzaz, Sawal H. B. M. Ali, Siti A. Ahmad, Mohd S. Islam and Javier E. (2015) "Selection of mother wavelet functions for multi-channel eeg signal analysis during a working memory task', Sensors, Vol. 15, pp. 29015-29035, November.

[18] Messer S. R., Agzarian J., Abbott D. (2001) "Optimal wavelet denoising for phonocardiograms", Microelectrons, Vol. 32, pp. 931-941, June.

[19] MohdTumari S. Z., Sudirman R., Ahmad A. H. (2013) "Selection of a suitable wavelet for cognitive memory using electroencephalograph signal", Engineering, Vol. 5, pp. 15-19. DOIi: $10.4236 /$ eng.2013.55B004.

[20] Lthotská L., Krajca V., mohylová J., Petránek S., Gerla v. (2009) "EEG data mining using PCA', Data Mining and Medical Knowledge Management: Cases and Applications, IGI Global, pp. 161180.

[21] Zaid Abdi., AlkareemAlyasseri., AhamadTajudi., Khader., Mohammed Azmi., Al-Betar "Electroencephagram Signals: Denoising using various Mother Wavelet Functions: A Comparative Analysis" ICISPC 2017, July 26-28 2017, Penang Malaysia

[22] Duo Chen ., Suiren Wan., Forest Sheng Bao “EEG based seizure Detection using Discrete Wavelet Transform through Full-level Decomposition” International Conference on BioInformatic and Biomedici (BIBM) 2015

[23] Ning Ji., Kaifeng Guo., Oluwarotimi Williams Samuel., Zhen Huang ;lisheng Xu ; and Guanglin $\mathrm{Li}$ "Appropriate mother wavelet for Continuous Gait Event Detection Based on time - frequency Analysis for Hemiplegic and healthy individuals". Sensor 2019

[24] Andrzejak R. G., Lehnertz K., Mormann F., Rieke C., David P. and Elger C. E. (2001) "Indications of nonlinear deterministic and finite-dimensional structures in time series of brain electrical activity: dependence on recording region and brain state', Physical Review E., Vol. 64, No. 6, Article ID 061907.

[25] Daubechies Ingrid (1992) “Ten lectures on wavelets", Industrial and Applied Mathematics, Philadelphia.

[26] Mallat S. (1989) “A theory for multi-resolution signal decomposition: the wavelet representation", IEEE Transaction on Analy. Mach. Intell., Vol. 11, No. 7, pp. 674-693.

[27] Rafiee J., Rafiee M., Prause N., Schoen M. (2011) "Wavelet basis functions in biomedical signal processing', Expert Systems and Applications, Vol. 38, pp. 6190-6201.

[28] Al-Kadi M. I., Reaz M., Ali M. (2012) "Compatibility of mother wavelet functions with the electroencephalographic signal', In Proceding of the 2012 IEEE EMBS Conference on Biomedical Engineering and Sciences (IECBES), Langkawi, Malaysia, pp. 113-117, 17-19 December.

[29] Halford J., R. Schalkoff and J. Zhou (2013) "Standardized database development for EEG epileptiform transient detection: EEGnet", Scoring system and machine learning analysis, Vol. 212 No. 2, pp. 308-316.

[30] Z. Haydari and H. Soltanian Zadeh (2011) "Semi automatic epilepsy spike detection from EEG signal using genetic algorithm and wavelet transform", IEEE int. conf. Bioinformatic Biomed. Work, pp 635-638 November.

[31] Subasi A., Gursoy M. I. (2010), “EEG signal classification using PCA, ICA, LDA and support vector machines”, Expert Systems with Applications, Vol. 37, pp. 8659-8666.

\section{Authors}

Atangana Romain was born in 1967 in Ngoumou-Cameroon. In 1997, he was graduated at the Higher technical Teacher's Training College (ENSET)University of Douala with DIPET 1 (bachelor's in Electrical and Electronics Engineering). In 2002 he obtained the DIPET 2 at the same institution. He obtained the DEA (Master of Sciences with Thesis) at the Ecole DoctoralePhysoique et Sciences de l'Ingénieur in Electronics, Electrotechnic, Automatic and Teledetection (EEAT) in 2007 at the University of DoualaCameroon. He is currently the Head of service of distance learning at the Higher

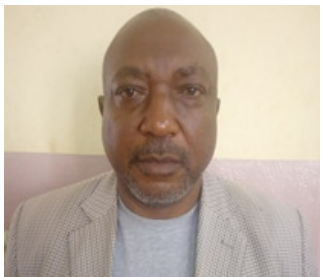
Teacher's Training College (ENS) of Bertoua-University of Ngaoundéré. In parallel to his job, he Mr. Atangana Romain is doing studies and research for a $\mathrm{PhD}$ thesis. His research interests are telemedicine, signal and image processing, artificial intelligence and automation, medical physics, and biomedical engineering. He is a member of the Unité de Recherche d'Automatique et d'InformatiqueAppliquée (UR- 
Signal \& Image Processing: An International Journal (SIPIJ) Vol.11, No.1, February 2020

AIA) of the IUT-FV of Bandjoun of the University of Dschang-Cameroon, and the Unité de Recherche de Matière Condensée et de Traitement de Signal (UR-MACETS) of the Faculty of Science of the University of Dschang-Cameroon.

Tchiotsop Daniel was born in 1965 in Tombel - Cameroon. He graduated in Electromechanicalengineering from the Ecole Nationale Supérieure Polytechnique (ENSP) of Yaoundé-Cameroon in 1990, he obtained a MS degree in Solid Physics in 1992 from the Faculty of Science of the University of Yaoundé I, a MS degree in Electrical Engineering and Telecommunication in 2003 from ENSP-Yaoundé and a PhD at INPL (Institut National Polytechnique de Lorraine), Nancy-France, in 2007. Dr TCHIOTSOP teaches in the Department of Electrical Engineering of the FOTSO Victor University Institute of Technology - University of Dschang

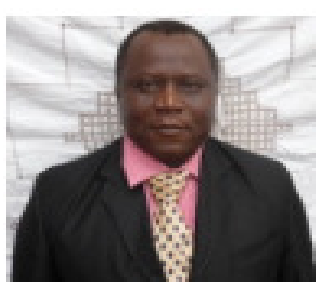
since 1999 where he is actually the Head of Department. He is with the Laboratoired'Automatique et d'InformatiqueAppliquée (LAIA) where his main items of research include Biomedical Engineering, Biomedical signal and image processing, Telemedicine and intelligent systems.Dr TCHIOTSOP ispartnerwith the Centre de Recherche en Automatique de Nancy (CRAN)-Université de Lorraine, France, Laboratoire d'Electronique et du Traitement de Signal (LETS)-ENSP, University of Yaoundé 1, and Unité de Recherche d'Automatique et d'Informatique Appliquée (UR-AIA) of the IUT-FV of Bandjoun of the University of Dschang-Cameroon.

KenneGodpromesse was born in Mbouda-Cameroon in 1967. He received the B.S. degree in electromechanical engineering and the M.S. degree in mechanical engineering from the Ecole Nationale Supérieure Polytechnique, Université de Taoundé I, Yaoundé-Cameroon in 1991 and 1994, respectively, and the $\mathrm{PhD}$ degree in control theory from the Université Paris XI, Orsay, France, in 2003. Since 1996, he has been with the Université de Dschang, Daschang-Cameroon, where he is currentl a Senior Lecturer. From 1996 to 2005, he has been the Head of the Department of Electrical Engineering, Fotso Victor University Institute of

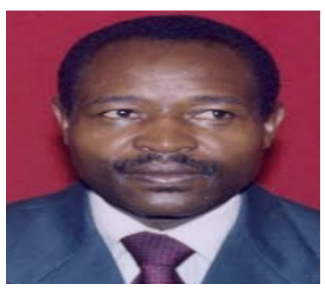
Technology,Bandjoun-Cameroon. He was a Guest Researcher at the Laboratoire des Signaux et Systèmes (L2S), Centre National de la Recherche Scientifique-L'Ecoled'Electricité(CNRS-SUPELEC), Université Paris XI, and the DépartementEnergie, SUPELEC, from September 2005 to January 2006 and from June to July 2006. Since April 206, he has been a founding of the Unité de Recherche d'Automatique et d'InformatiqueAppliquée (UR-AIA) of the IUT-FV of Bandjoun of the University of Dschang-Cameroon. His current research interests include identification and control of nonlinear systems using variable structure and neural network with applications in electromechanical systems.

DjoufackNkengfack Laurent Chanel was born in 1991 in Nanga-EbokoCameroon. In 2014, he obtained the Bachelor degree in Physics option Electronics, Electrotechnic and Automatic at the University of DschangCameroun. In 2016 he was graduated at the same institution with the Master of Sciences with thesis in Physics option Electronics, Electrotechnic and Automatic. In 2018 he obtained the DIPET 2 in Electrical Engineering option Electronics at the Higher Teacher's Training College of Bambili-University of Bamenda-

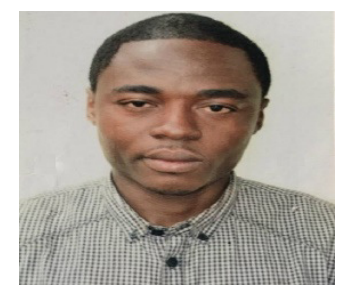
Cameroon. He is currently a Technical High School teacher in electronics engineering. In parallel to his job, Mr. Djoufack is doing studies and research for a $\mathrm{PhD}$ thesis. His research interests are telemedicine, signal and image processing, artificial intelligence, medical physics, and biomedical engineering. He is a member of the Unité de Recherche d'Automatique et d'InformatiqueAppliquée (UR-AIA) of the IUT-FV of Bandjoun of the University of Dschang-Cameroon, and the Unité de Recherche de Matière Condensée et de Traitement de Signal (UR-MACETS) of the Faculty of Science of the University of Dschang-Cameroon. 